\title{
CENTRAL NERVOUS SYSTEMS' EFFECTS OF ISOFLURANE (FORANE)
}

\author{
Eva M. Kavan, M.D. and Robert M. Julien, Ph.D.
}

\section{INTRODUCTION}

Isoflurane (Forane, ${ }^{\circ}$ 1-chloro-2,2,2,-trifluoroethyl difluoromethyl ether (is a recently developed short-chain halogenated inhalation agent. It is an isomer of enflurane, with a different boiling point and vapour pressure. ${ }^{1}$ Isoflurane provides rapid induction as well as prompt emergence from anaesthesia. Isoflurane-induced anaesthesia has been investigated in experimental animals ${ }^{2-4}$ and in man. ${ }^{5-8}$ Its effects on the cortical EEG have been determined in dogs, ${ }^{4}$ in human volunteers ${ }^{7}$ and in patients during operations. ${ }^{8}$ However, the effects of isoflurane on subcortical structures have not been investigated. The present study was conducted to supply these data.

\section{METHODS}

\section{A. Chronic Experiments}

Five cats (average weight $4.8 \mathrm{~kg}$ ) were used in eight experiments. Under pentobarbital anaesthesia, using sterile technique, bipolar concentric stainless steel electrodes ( $0.2 \mathrm{~mm}$ diameter) were implanted stereotaxically ${ }^{9}$ into the following structures: n. caudatus (CAUD), n. ventralis postero-lateralis (primary relay nucleus) of the thalamus (VPL), the midbrain reticular formation ( $R F$ ), $n$. centrum medianum (CM), n. amygdalae (AMYG) and formatio hippocampi (HIPP). Stainless steel screws were imbedded in the skull over frontal and parietal cortices. Leads from all electrodes were soldered to miniature Winchester sockets which were secured to the skull with acrylic resin.

Two weeks after implantation, the cats were taken to the laboratory for conditioning and control tracings. In the following week, isoflurane vapourized through a copper kettle was administered by mask, using a total of $1 \mathrm{~L} / \mathrm{min}$ flow of equal parts of air and oxygen in a semiclosed system. End-tidal carbon dioxide was monitored with a Beckman infrared gas analyser by a \#19 polyethylene catheter inserted into one nostril. $\mathrm{CO}$.2 was recorded continuously together with cortical and subcortical activity on an 8-channel Grass polygraph.

In the first group of experiments (Figure 1) isoflurane was administered initially in a low concentration ( 2.4 per cent) and when the changes observed in the brain activity became stabilized, the concentration was increased to 4.7 per cent and 6.9 per cent respectively. In a second group of experiments, a rapid induction was

From Department of Medical Pharmacology \& Therapeutics, California College of Medicine, University of California, Irvine, Irvine, California 92664

Supported by a grant from the Rebecca Payne Livingston Foundation.

-Trademark of Ohio Medical Products, a Division of Airco, Inc. 


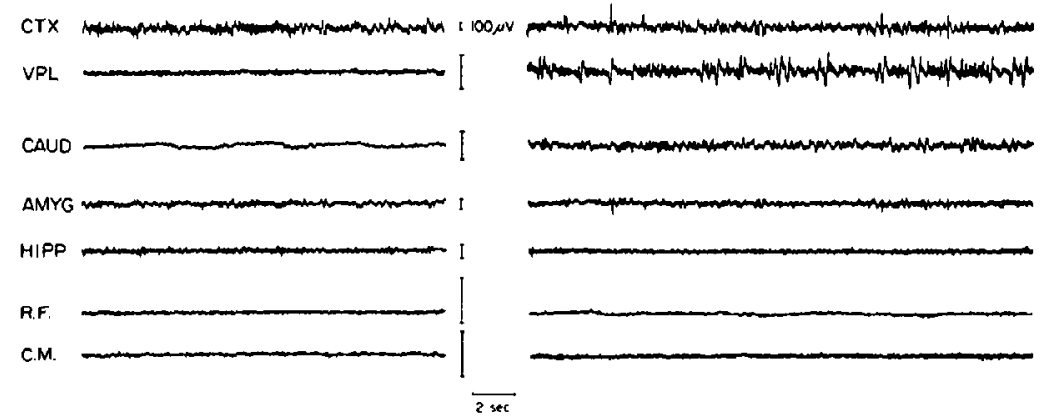

C
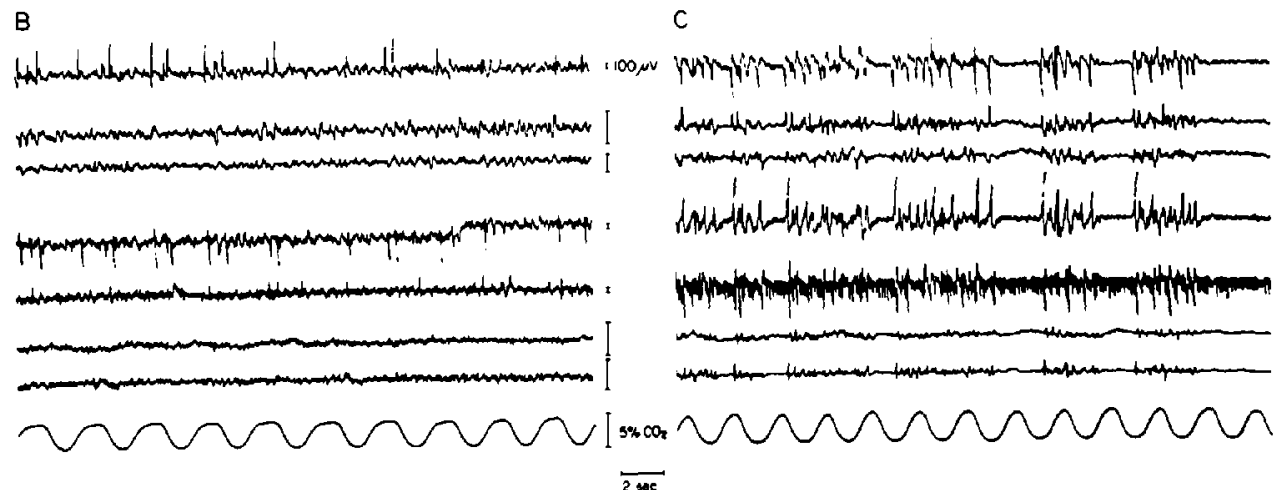

Figure 1. Spontaneous EEG recorded during slow induction of anaestheisia with isoflurane; $A=2.4$ per cent concentration administered for about 2 minutes. Cat quiet but alert. $B=$ about 2 minutes of 4.7 per cent. $C=$ about 1 minute of 6.9 per cent, animal unresponsive $\left(P^{-}\right)$. Eighth line, end-tidal $\mathrm{CO}$... Same calibration for control and A (upper tracings) and reduced for $\mathrm{B}$ and $\mathrm{C}$ (lower tracings), as high voltage activity developed. Abbreviations: $\mathrm{CTX}=$ frontal cortex, $\mathrm{VPL}=\mathrm{n}$. ventralis posterolateralis, $\mathrm{CAUD} .=\mathrm{n}$. caudatus, $\mathrm{AMYG} .=\mathrm{n}$. amygdalae, $\mathrm{HIPP} .=$ formatio hippocampalis, $\mathrm{RF}=$ midbrain reticular formation, $\mathrm{CM}=\mathrm{n}$. centrum medianum.

followed by a brief administration of 6.9 per cent. With both types of induction, as soon as the cats appeared to have lost consciousness (i.e. were no longer moving spontaneously), one hind leg was stretched and the paw was pinched with a clamp at irregular intervals. As soon as the animal lost reflex withdrawal to this noxious stimulus (i.e. attained anaesthesia), the concentration of isoflurane was gradually decreased to the lowest concentration needed, and maintained for $30 \mathrm{~min}$. The brain activity was continuously monitored. At the end of 30 minutes, isoflurane was discontinued and the EEG was monitored until the cats were behaviourly awake and again moving spontaneously. The animals were observed in the laboratory for the remainder of the day. Tracings were obtained at two and four hours after the end of the experiment and on the first, second, fourth and seventh days after anaesthesia.

\section{B. Acute Experiments}

Eight cats (average weight $4.2 \mathrm{~kg}$ ) were utilized. A tracheotomy was performed under ether anaesthesia and the femoral artery and vein were cannulated for direct blood pressure measurements and drug administration respectively. The animals 
were mounted in a stereotaxic frame, the scalp retracted and burr holes drilled to permit introduction of electrodes into subcortical structures. Two stainless steel electrodes were inserted into the subcutaneous tissue of each forelimb for somatic stimulation and additional needles for recording of the EEG. When all painful procedures were completed, the exposed wound margins were infiltrated with lidocaine. The animals were immobilized by intermittent intravenous doses of gallamine and were artificially ventilated at a rate of $25 / \mathrm{min}$ with a tidal volume adjusted in each animal to give end-tidal $\mathrm{CO}_{2}$ of 4 per cent. A \#19 polyethylene catheter was inserted into the tracheostomy tube and connected to a Beckman LB-1 gas analyzer for monitoring of the end-tidal $\mathrm{CO}_{2}$. Subcutaneous temperature was monitored by a probe inserted subcutaneously in the lower abdomen, and maintained at $38^{\circ} \mathrm{C}$ by moderate heating.

The cortical activity was determined by use of a stainless steel needle implanted into the skull over the somato-sensory area $\left(S_{1}\right)$ while the indifferent electrode (a stainless steel screw) was fastened over the frontal sinus. Bipolar concentric stainless steel electrodes insulated on the outside except for the very tip ( $50 \mu$ tip diameter) were used to record spontaneous and evoked activity in subcortical structures. The electrodes were introduced stereotaxically into the specific somatic relay nucleus of the thalamus ( $n$. ventralis postero-lateralis, VPL), into nucleus centrum medianum (CM) of the non-specific thalamic system, and into the midbrain reticular formation $(\mathrm{RF})$.

Peripheral stimulation consisted of single shocks ( 8 volts intensity, $0.2 \mathrm{msec}$ duration ), applied every four or six seconds. Stimulation of the contralateral forelimb was used to elicit responses in the sensory-motor cortex $\left(S_{1}\right)$ and in VPL. Both ipsilateral and contralateral stimulation were used for responses recorded from the RF and the CM. The evoked cortical and subcortical potentials were amplified, displayed on an oscilloscope and photographed. In addition, twenty subsequent responses were recorded and averaged in a Computer of Average Transients.

Control tracings of evoked potentials in all structures were obtained not less than three hours after the termination of general anaesthesia which was used for the preparatory surgery, to assure complete recovery from the effects of ether. Isoflurane was vapourized in a copper kettle and then administered in a $1 / 1$ airoxygen mixture starting with a low ( 2.4 per cent) concentration which was gradually increased to 4.7 per cent. The evoked responses were photographed and averaged with each change of concentration of isoflurane, and during recovery from the anaesthetic.

Placement of electrodes in subcortical structures was verified histologically both in the chronic preparations (when the animals were sacrificed at the end of the study) and at the end of each acute experiment.

\section{Chronic Experiments}

\section{Results}

The first effects of low concentrations of isoflurane ( 2.4 per cent) were observed in the VPL and caudate (Figure 1a). There was a marked increase in amplitude, more pronounced in the caudate, with simultaneous reduction in frequency. There 
were occasional spikes. The next alterations, consisting also of increased amplitude but to a lesser extent were seen in the cortex and $n$. amygdalae. In some experiments in both these latter structures, the amplitude alternately increased and decreased, forming a type of spindle similar to those seen in barbiturate-induced sleep. The frequency of the electrographic activity from the four structures was reduced from the fast $(30 \mathrm{~Hz})$ activity characteristic of wakefulness to 12 to $16 \mathrm{~Hz}$. The cats became progressively more quiet, but resisted when pinched and they did not tolerate insertion of the polyethylene catheter into the nostril.

An increase of Forane concentration to 4.7 per cent (Figure 1b) was followed in CTX, VPL, and AMYG by further increases in amplitude and by further slowing. Simultaneously, synchronous spikes appeared in the cortex and amygdala. Slight increases in amplitude with slowing and occasional spikes appeared in the hippocampus. At this point, animals lost consciousness but still moved in response to pinch of the hind paw.

With a further increase of concentration of isoflurane to 6.9 per cent, the spikes became more frequent in the limbic structures and in the cortex, while in the VPL and caudate nuclei the high voltage activity became still slower (continuous hypersynchrony). Most animals no longer withdrew their limbs in response to a stretch and hard pinch of the hind paw. EEG activity was stabilized with continuous hypersynchrony and spikes. The concentration of isoflurane could then be decreased to between 3 and 4 per cent for maintenance of anaesthesia (characterized as nonresponsiveness to a noxious stimulus) and these electrographic patterns remained stable.

In some experiments, however (Figure 1c), the cats were still responding to a pinch of the hind paw during the period of continuous hypersynchrony. Further administration of isoflurane, in the same concentration (6.9 per cent), resulted in additional changes in the brain activity. The hypersynchronous activity developed in all structures with higher frequency spiking in the hippocampus. This activity then became interrupted by brief intervals of low voltage $(20 \mu \mathrm{V})$, fast $(30 \mathrm{~Hz})$ activity (intermittent hypersynchrony, Figure 1c). These episodes were at first of very short duration ( 2 seconds or less) but gradually increased to 10 seconds. The periods of hypersynchrony varied between three and six seconds. These hypersynchronous bursts were also observed in the RF and CM, two structures which so far were only very little affected. At this point all animals lost reflex withdrawal to stretching and pinch of the hind paw and the concentration of isoflurane could be reduced to 3 or 4 per cent. During the following 30 minutes while administration of isoflurane continued with a concentration needed for maintenance of anaesthesia, the pattern of intermittent hypersynchrony regressed to a pattern of continuous hypersynchrony.

The changes described above were observed during deliberately slow induction, i.e. the initial concentration of isoflurane was low ( 2.4 per cent) and never increased unless the changes in the brain activity were well established and stabilized (Figures $1 \mathrm{a}, 1 \mathrm{~b}, 1 \mathrm{c})$. It is common practice to reduce induction time by administering anaesthetics initially in a high concentration. Therefore, in a few experiments isoflurane was administered with a higher starting concentration (4.7 per cent) followed by fairly rapid increase to 6.9 per cent. These electrographic changes are 


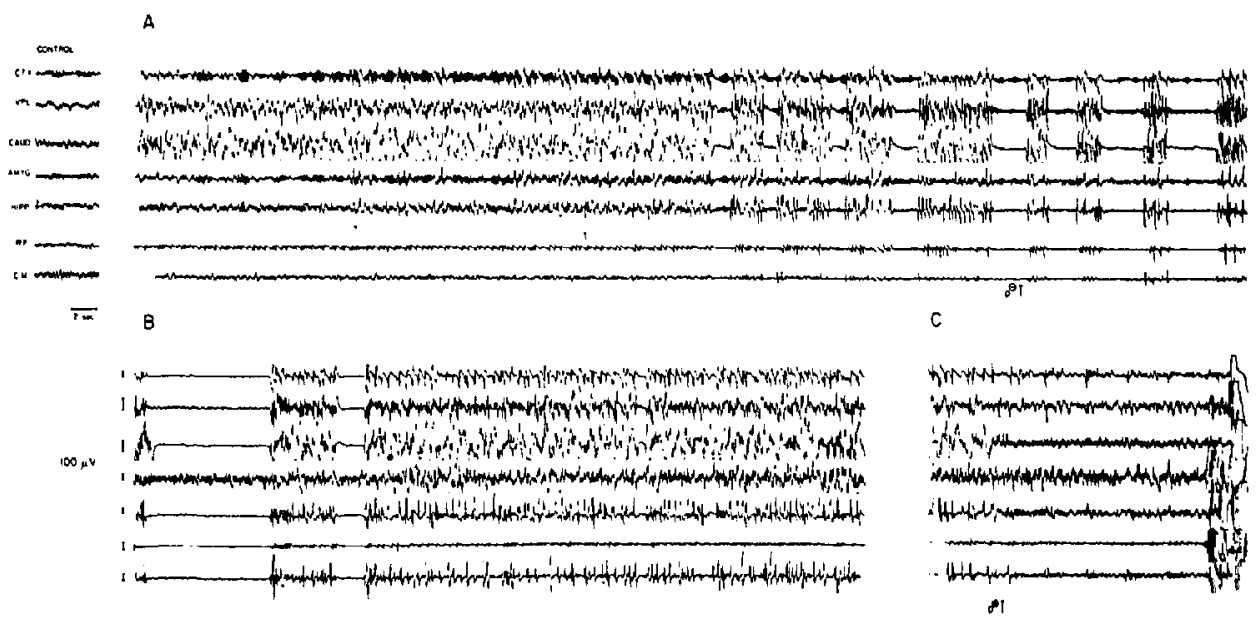

FIgure 2. Spontaneous EEG activity during rapid induction of isoflurane anaesthesia. A. Initial concentration 4.7 per cent. First arrow end of the first minute; note onset of hypersynchrony in VPL and caudate nuclei and high voltage spikes in cortex, n. amygdalae and hippocampus; the cat is quiet but still responds to pinch. At the second arrow, concentration increased to 6.9 per cent; note transition of continuous hypersynchrony to intermittent hypersynchrony, followed in about 30 seconds by lack of response to a similar stimulus ( $\mathrm{P}-$, arrow lower right). Concentration of isoflurane was reduced at this point to a maintenance concentration of 3.6 per cent. B. Maintenance with 3.6 per cent for about 2 minutes. C. Isofhurane discontinued (after 30 minutes of maintenance) about 20 seconds before left margin of tracing; note rapid return toward control appearance with simultaneous positive response to a pinch $(\mathrm{P}+)$. Artefact at extreme right created by spontaneous head movement. Abbreviations same as in Figure 1.

illustrated in Figure 2. As may be seen, the EEG alterations resembled those of Figure 1 but developed much more rapidly. Loss of reflex withdrawal to pinch occurred concomitant with periods of intermittent hypersynchrony (Figure 2a, right side) and the unresponsiveness could be maintained by decreasing the concentration of isoflurane to that necessary to maintain the EEG in a state of continuous hypersynchrony (Figure $2 b$ ) , 3 to 4 per cent.

When inhalation of isoflurane was terminated (Figure 2c), emergence from the anaesthetic was extremely rapid. Within 30 to 60 seconds the animals were behaviourally awake and the EEG patterns reverted promptly to control. Frequently the hippocampus and amygdala were the last to recover. The tracings taken at 1 hour and 2 hours after discontinuation of isoflurane resembled control tracings. In the 4-hour record higher amplitude and occasional slowing were observed in the cortex, CM, VPL and caudate. Similar but less pronounced changes were seen in the 24-hour record. No residual effects were observed in records taken at four and seven days after the experiment.

Inhalation of isoflurane did not appear to irritate the upper respiratory pathways and excessive salivation was not observed. Respiration was adequate both by clinical observation and by objective measurements of end-tidal $\mathrm{CO}_{2}$ which remained constant in the vicinity of 4 per cent (Figures $\mathrm{lb}$ and $1 \mathrm{c}$ ). Pupils were never dilated.

Muscle twitching and abnormal movements were not seen during any of these experiments and there was no ataxia or shivering during recovery. 
S-M CTX
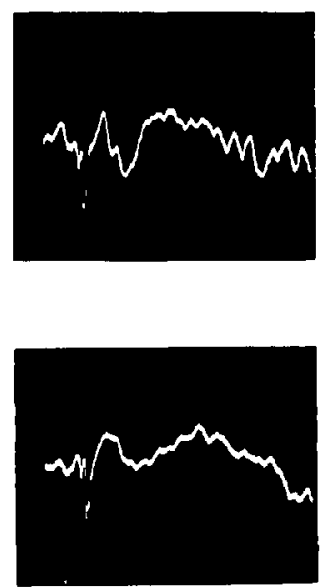

RECOVERY
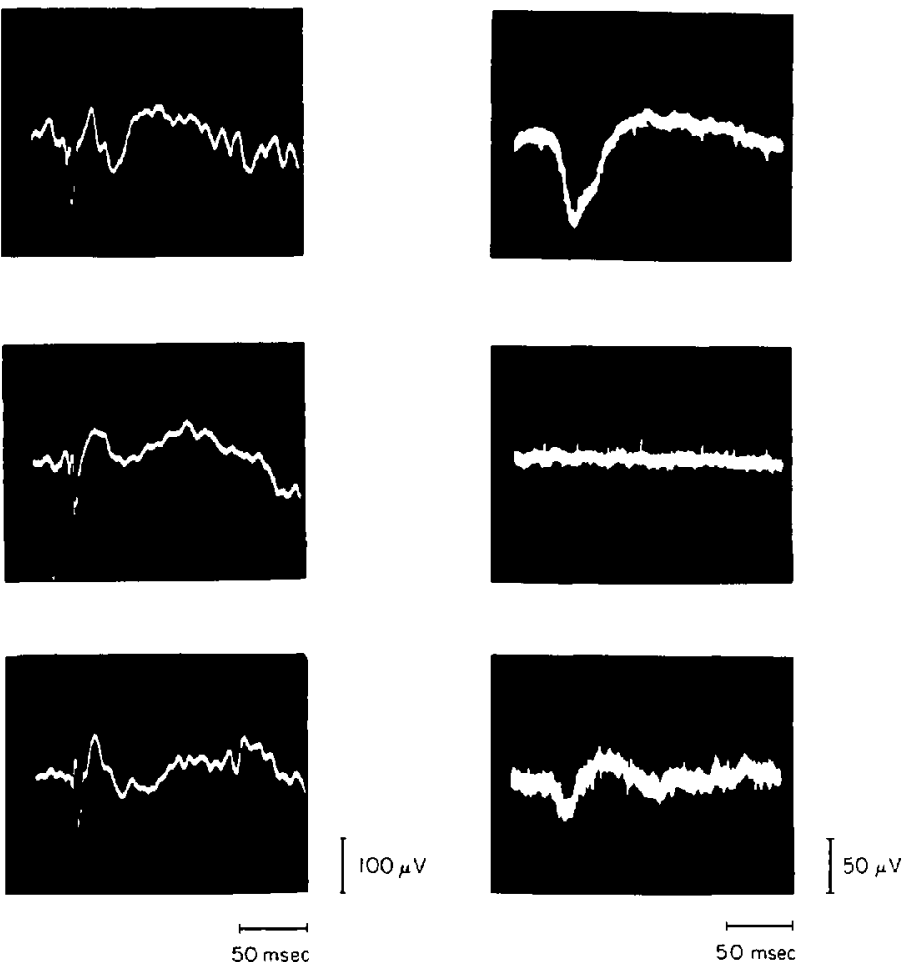

Figure 3. Evoked potentials elicited by a single pulse $(8 \mathrm{~V}, 0.2 \mathrm{msec})$ to contralateral forepaw and recorded in the sensory-mator cortex (S-M ctx) (left column) and in midbrain reticular formation (RF) in response to ipsilateral forepaw stimulation (right column). Upper tracing before administration of isoflurane, middle tracing during inhalation of 2.4 per cent of isoflurane and lower tracing 30 minutes after Forane was discontinued.

\section{Acute Experiments}

In experiments on acutely prepared animals, responses evoked by peripheral stimulation were analyzed before and after administration of isoflurane. After recording of control responses, isoflurane was administered in a low (2.4 per cent) concentration. At least three minutes were allowed for equilibrium before subsequent stimuli were applied. Evoked responses were first recorded in the two structures of the non-specific sensory system (RF and $\mathrm{CM}$ ) repeated 20 times at 6second intervals for each structure and averaged. Stimuli were then applied at somewhat shorter intervals (4 seconds) and evoked responses were recorded in the cortex and VPL. The total time needed for this sequence of recordings at 2.4 per cent isoflurane was approximately 15 minutes. Spontaneous cortical activity which was monitored simultaneously underwent alterations closely resembling those observed in chronic preparations. During this 15-minute period of isoflurane administration, spontaneous cortical activity developed to a slow, high voltage pattern with spikes (Figure 5, second line). At a higher concentration of isoflurane ( 4.7 per cent), hypersynchrony gradually developed and became intermittent (burst suppressions, Figure 5, third line) in a manner closely resembling that pro- 
A CONTROL

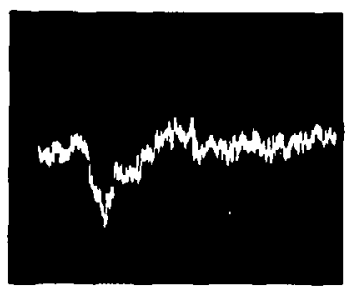

C 10 mIn RECOVERY

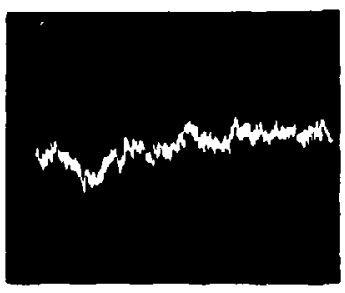

B FORANE $3 \mathrm{~min}$

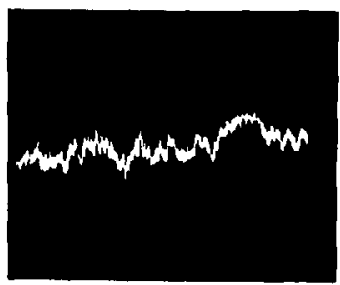

E $20^{\prime}$ RECOVERY

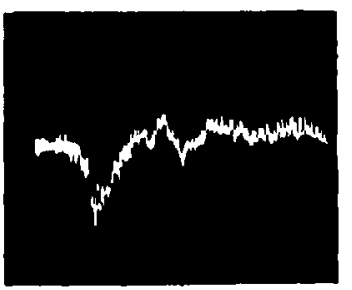

C FORANE $15 \mathrm{~min}$

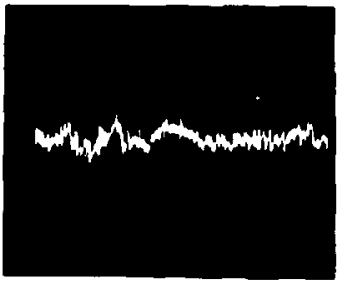

F $30 \mathrm{~min}$ RECOVERY

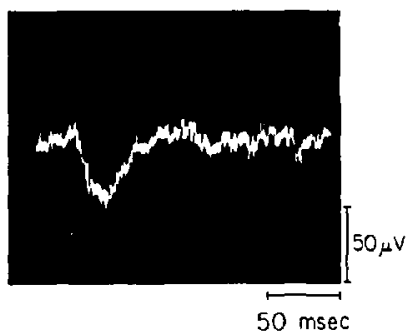

Ficure 4. Evoked potentials recorded in $\mathrm{CM}$ following single ipsilateral forepaw stimulation $(8 \mathrm{~V}, 0.2 \mathrm{msec}$ ) before (A), after 2.4 per cent isoflurane (B), after 4.7 per cent isoflurane (C), and during recovery (D-F).

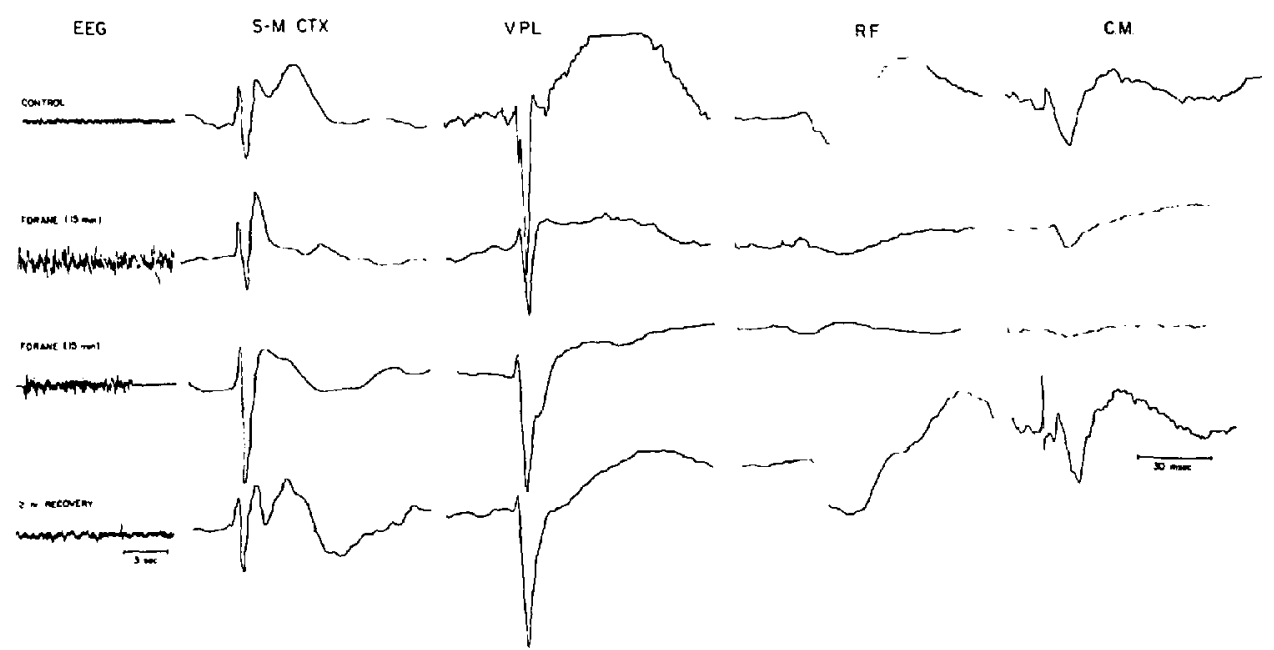

Ficune 5. Effect of isoflurane on spontaneous EEG and evoked cortical and subcortical responses in acute animals. Left column = EEG before isoflurane, after 15 minutes of 2.4 per cent isoflurane, after 15 minutes of 4.7 and after two hours of recovery. Remaining four columns $=$ computer averages of 20 responses to forepaw stimulation recorded in contralateral $S-M$ ctx and $\mathrm{VPL}$ and in ipsilateral RF and CM.

duced by a concentration of 6.9 per cent administered by face mask to the animals with chronically implanted electrodes (Figure lc).

\section{Evoked Responses}

1. Sensory-Motor Cortex. In most experiments the latency and amplitude of the negative and positive components of the responses evoked by stimulation of 
the contralateral forepaw were unchanged (Figure 3, left), regardless of the concentration of isoflurane inhaled. In some instances the positive phase was smaller at first but became much larger when higher concentrations were used (Figure 4). On the other hand, there was occasionally an initial increase in amplitude of the positive component with a subsequent return to the control appearance during recovery. Long latency slow waves were never observed during the administration of isoflurane.

2. N. Ventralis Postero-lateralis of Thalamus (VPL). In the majority of experiments there were no changes in either the latency or the amplitude of the responses evoked by stimulation of the contra-lateral forelimb. In a few instances, the amplitude slightly decreased with 2.4 per cent isoflurane but increased again with the higher concentration (Figure 5 ).

3. Reticular Formation. With low concentrations of isollurane (2.4 per cent) the latency of response of the RF elicited by stimulation of the contralateral or ipsi-lateral forelimb remained unchanged, but the amplitude was greatly decreased or abolished (Figures 3 and 5) within minutes after inhalation of isoflurane was started. Recovery was relatively slow, but usually complete within 30 to 120 minutes (Figures 3 and 5 ).

4. N. Centrum Medianum (CM). Low concentrations of isoflurane ( 2.4 per cent) depressed or abolished evoked responses in the CM following either ispi-lateral or contra-lateral stimulation, in a manner similar to that observed in the RF (Figures 4 and 5). The latency of the response was not altered. In some experiments, higher concentrations ( 4.7 per cent) of isoflurane were needed to totally abolish evoked responses (Figure 5). Recovery was comparable to that for the $\mathrm{RF}$.

There were no changes in heart rate or end-tidal $\mathrm{CO}_{2}$ and only minimal deviations in arterial blood pressure during these experiments. In only a few instances, a 20 torr decrease in systolic pressure was noticed with the 4.7 per cent concentrations of isoflurane.

\section{Discussion}

In experiments on animals with chronically implanted electrodes the effect of new anaesthetic agents on brain activity can be investigated under conditions which resemble clinical investigations to a certain extent. Control tracings may be taken before administration of the drug, through induction and maintenance and finally during recovery emergence. However, there are certain differences which should be recognized. For example, the concentration of isoflurane used in our experiments had to be considerably higher than reported for human subjects. ${ }^{6,8}$. The reasons for this are several. First, it is well known that the concentrations of volatile anaesthetics needed to attain anaesthesia in cats are higher than those required for man. ${ }^{10}$ Also, the animals are not premedicated before the experiment nor are they intubated, the nitrous oxide used in clinical anaesthesia is replaced by air in order to avoid effects of any drug other than the agent under investigation. Third, there is considerable dead space in the mask which is essentially just a cone and is not contour fitted as are patients' masks. This mechanical dead space is eliminated in acute experiments where the agent is administered through the 
respirator with a hose connecting the inspiratory arm of the ventilator directly to the tracheostomy tube.

This difference in anaesthetic technique and apparatus in chronic animals (isoflurane delivered by mask) and in acute animals (isoflurane delivered into the tracheal cannula) explains why the $\mathrm{P}^{-}$state and intermittent hypersynchrony were achieved with 6.9 per cent isoflurane in chronic animals but with only 4.7 per cent in acute experiments.

This difference in isoflurane concentration is not critical, however, since it was not the objective of these experiments to delineate definite EEG patterns coinciding with various depths of anaesthesia. The electrographic changes which we describe are observations from the very onset of inhalation of isoflurane, through induction and until non-responsiveness to a noxious stimulus (i.e. light anaesthesia) is attained. Similar criteria were used by Merkel. ${ }^{11}$ In modern clinical practice deeper levels of anaesthesia are avoided because muscular relaxation is obtained by use of muscle relaxants. Although pinching of a hind paw is not comparable to the stimulus provided by surgical incision, it is presently accepted for determination of minimal anaesthetic alveolar concentrations (MAC).$^{11,12}$ We have maintained this level of anaesthesia for 30 minutes before discontinuing inhalation of the agent. Indeed, except for the rapid-induction experiments (Figure 2), each concentration of isoflurane was administered for as long as was necessary for EEG patterns to stabilize, reflecting an equilibrium in the brain. The patterns were correlated with the animal's behaviour. No attempt was made to correlate any given pattern with any specific alveolar concentration. During induction this would have been technically impossible. For example, with a low ( 2.4 per cent) concentration of isoflurane the cats would not permit insertion of a catheter into their nostrils since, although not vigorously resisting the mask, they had not lost consciousness. Once anaesthetized, however, the catheter could be inserted for the monitoring of expired $\mathrm{CO}_{2}$ (Figures $1 \mathrm{~b}, 1 \mathrm{c}$ ).

Observations of changes occurring in cortical activity at increasing depth of anaesthesia are usually included in investigations of new anaesthetic agents. For isoflurane, these alterations were reported both in experimental animals and in man. ${ }^{4,7,8}$ The effects on subcortical structures have not been studied. Likewise, mechanisms by which anaesthesia is produced in the brain are not known but there is increasing evidence that subcortical structures are involved. It is felt that investigations of the effect of a new anaesthetic on the central nervous system are not complete unless the subcortical effects of the drug are also investigated. Observations should be conducted from the beginning of administration of the agent, during maintenance and recovery and eventually in post-anaesthesia hours and days. For this purpose a chronic preparation is ideally suited. A large number of electrodes can be implanted over the cortex and in several subcortical structures. The simultaneous use of more than a very few electrodes is technically impossible in an acute preparation. Therefore recording electrodes were permanently implanted in two representative structures of each of the three systems currently thought to participate in the anaesthetic state (limbic, somato-sensory and non-specific sensory systems). ${ }^{13}$

Initial changes, after only a brief inhalation of low concentration of isoflurane 
2.4 per cent) occurred in the subcortical sensory structures (the VPL and caudate nuclei), followed by changes in the cortex and limbic structures, while the structures of the non-specific sensory system (CM and RF) remained essentially unaltered. Alterations in subcortical structures occurred before consciousness was lost.

In our previous report ${ }^{10}$ of the effects of volatile anaesthetics on the sensory and limbic systems, we have observed that with fluorinated agents (halothane, methoxyflurane and enflurane) the first alterations of EEG patterns occurred in the limbic structures, while with ether, earliest changes occurred in the cortex and VPL. Thus, the electrographic effects of isoflurane (a fluorinated ether) resemble diethyl ether more than other fluorinated compounds.

With isoflurane, onset of anaesthesia was accompanied by either continuous hypersynchrony in all structures, or, in about an equal number of experiments, by intermittent hypersynchrony separated by brief periods of low voltage fast activity. Hypersynchrony was observed previously with methoxyflurane, but only after anaesthesia had been maintained for at least 15 to 20 minutes. ${ }^{10}$ Continuous hypersynchrony was never observed with halothane, although frequent spikes were prevalent during maintenance of anaesthesia. With enflurane, an isomer of isoflurane, frequent spikes occurred almost simultaneously in all structures monitored in our experiments, while the animals still responded reflexly to pinching. The spikes were not grouped as regularly as with isofurane but rather appeared in a pattern similar to idiopathic epileptic seizures. Groups of spikes of uneven amplitudes were separated by brief and later prolonged periods of isoelectricity. This type of pattern was typical for anaesthesia with enflurane. If the concentration of enflurane was decreased for maintenance, burst suppressions reverted to continuous hypersynchrony, but simultaneously anaesthesia was lost. ${ }^{15} \mathrm{With}$ isoflurane, anaesthesia was maintained while patterns of hypersynchrony dominated the records.

There is another important finding, peculiar to isoflurane. The groups of high voltage activity (intermittent hypersynchrony) were never separated by electrical silence as was observed with its isomer, enflurane. The fast, low voltage activity (Figure 1c) could better be compared to the patterns of arousal. This would exclude the possibility that we are describing unintentionally deep levels of anaesthesia or a certain degree of hypoxia due to respiratory depression (verified by $\mathrm{CO}_{2}$ tracing in Figures $\mathrm{lb}$ and $1 \mathrm{c}$ ).

Summarizing the comparison of the effect of isoflurane on the brain with the effects of other fluorinated agents, we feel that we have to qualify our previous statement, that the limbic structures are first affected, because with isoflurane the first alterations were observed in the VPL, caudate and cortex as seen with ether. With halothane and methoxyflurane no definite pattern was consistent with onset of anaesthesia, with isoflurane the cats ceased to respond to pinching of the hind paw concomitant with patterns of continuous or intermittent hypersynchrony. With enflurane, anaesthesia was achieved and maintained only while high voltage spiking alternated with isoelectricity.

With all four fluorinated agents, occasional (halothane), frequent (methoxyflurane), or continuous spikes (isoflurane), or spikes separated by burst suppressions (enflurane) are consistent with maintenance of anaesthesia in cats. 
Although it is technically possible in chronic preparations to record potentials evoked by peripheral sensory stimulation, acute experiments are better suited for this type of study. The disadvantage of acute experiments is that the animals are paralyzed and unable to respond to a noxious stimulus by spontaneous movement. However, this disadvantage can be overcome by the simultaneous monitoring of spontaneous cortical EEG activity and comparing these patterns with those observed in chronic preparations. The difference in the administration of isoflurane in these two types of experiments makes any comparison only very limited. In chronic experiments the animals are breathing spontaneously through a mask, which includes a relatively large dead space. This is eliminated in acute experiments by the direct connection of the ventilator to the tracheostomy tube. In addition, in acute preparations isoflurane was administered by positive pressure and each concentration was administered for a considerably longer period. We have observed that more profound alterations in the spontaneous brain activity occurred at the end of 13 to 15 minutes with comparatively lower concentration of the agent.

It is generally recognized that potentials evoked along the oligosynaptic specific sensory pathways persist during general anaesthesia, although they might be slightly altered in amplitude or configuration. ${ }^{14}$ Our own observations on the structures of somato-sensory pathways and Joas, et al. ${ }^{4}$ in auditory evoked potentials confirmed this to be true with inhalation of isoflurane.

The multisynaptic non-specific sensory system is particularly susceptible to the effects of hypnotic and anaesthetic agents. ${ }^{14}$ This susceptibility may be manifested either by depression of the evoked potentials recorded in structures comprising this system or by facilitation of the responses. ${ }^{1+}$ Barbiturates and most volatile agents depress or abolish the evoked activity while chloralose, hydroxydione ${ }^{14}$ and enflurane $^{15}$ induce facilitation. Isoflurane markedly depressed evoked potentials in the non-specific structures explored in our experiments (Figures 3-5).

An interesting observation was made during the recording of evoked potentials in the VPL. With the higher concentration of isoflurane (4.5 per cent) the evoked response reversed in polarity. It was suspected that this reversal indicated a considerable change of relationship between the brain tissue and the recording electrode. Responses were subsequently recorded bilaterally and as soon as the response reversed, one electrode was kept stationary and the inverted response was recorded as long as isoflurane was administered. The electrode on the opposite side was advanced until the original shape of the response could again be recorded. During recovery only the stationary electrode recorded a normal response while the electrode which had been further advanced had to be withdrawn 1 to $2 \mathrm{~mm}$ in order to obtain an identical pattern. This indicated indirectly that the brain volume was temporarily reduced. Since there were no changes in the end-tidal $\mathrm{CO}_{2}$ throughout the experiment this observation cannot be attributed to the effect of hyperventilation. Direct measurements are needed to confirm this observation.

\section{SUMMARY}

Electrographic effects of isoflurane (Forane) on the CNS were evaluated in chronic and acute experiments. In cats with chronically implanted electrodes, EEG 
activity was recorded from the cortex and from selected subcortical structures. First changes during inductions of anaesthesia were observed in the structures of the specific somato-sensory system, followed by high voltage spikes in the limbic structures. Continuous hypersynchrony in all leads developed simultaneously with onset of anaesthesia and persisted or developed further into intermittent hypersynchrony during maintenance. Upon termination of inhalation of isoflurane, return to control EEG patterns and behavioural arousal occurred within seconds. In acute preparations, evoked responses recorded in the specific somato-sensory pathways following forepaw stimulation were only slightly affected, but such responses were markedly depressed or abolished in the diffuse reticulo-thalamic activating system.

\section{RÉSUMÉ}

On a pu évaluer les changements électrographiques de l'isoflurane (forane) sur le S.N.C. A partir d'électrodes implantées dans le cerveau de chats, on a enregistré l'activité électro-encéphalographique venant du cortex et des structures sous corticales. Au moment de l'induction de l'anesthésie on a vu apparaître les premières modifications dans le système somato-sensoriel, suivies de pointes à haut voltage au niveau du système limbique. Le début de l'anesthésie a montré un hypersynchronisme dans toutes les dérivations et qui a persisté ou s'est modifié en un hypersynchronisme intermittent durant le maintien de l'anesthésie. Après avoir cessé l'administration d'isoflurance on est revenu rapidement au tracé électroencéphalographique de l'éveil. Dans les préparations d'animaux, chez qui les électrodes venaient d'être implantées, les réponses obtenucs venant des voies somato-sensorielles et produites par la stimulation des membres antéricures ont été peu modifiées; toutefois elles étaient profondément déprimées voire mème abolies au niveau du système réticulo-thalamique activateur.

\section{ACKNOWLEDGMENTS}

We would like to thank D.M. Kunis, B.A., for skillful technical assistance.

We are grateful to Mr. J. Vitcha (Ohio Medical Products) a Division of Airco, Inc. for the generous supply of isoflurane used in these experiments.

\section{REFERENCES}

1. Viтcha, J.F. A history of Forane. Anesthesiology 35: 4-7 (1971).

2. Byles, P.H., Dobkin, A.B., Fercuson, J.H., et al. Forane (compound 469) : 2. Biochemical effects of repeated administration to animals, response to bleeding and compatibility with epinephrine. Can. Anaesth. Soc. J. 18: 387-396 (1971).

3. Byles, P.H., Dobkin, A.B., \& Jones, D.B. Forne (compound 469): 3. Comparative effects of prolonged anaesthesia on mature beagle dogs and young Rhesus monkeys. Can. Anaesth. Soc. J. 18: 397-407 (1971).

4. Joas, T.A., Stevens, W.C., Eger, E.I., II Electroencephalographic seizure activity in dogs during anaesthesia. Brit. J. Anaesth. 43: 739-745 (1971).

5. Cromper.h, T.H., Eger, E.I., II, Stevens, W.C., et al. Forane uptake, excretion, and blood solubility in man. Anesthesiology 35: 401 (1971).

6. Donkin, A.B., Byles, P.H., Ghanooni, S., et al. Clinical and laboratory evaluation of a 
new inhalation anaesthetic: Forane (compound 469) $\mathrm{CHF}_{2}-\mathrm{O}-\mathrm{CHClCF}_{2}$. Can. Anaesth. Soc. J. 18: 264-271 (1971).

7. Eger, E.I., II, Stevens, W.C., Cromweld, T.H. The electroencephalogram in man anesthetized with Forane. Anesthesiology 35: 504-508 (1971).

8. Homi, J., Konchiceri, H.N., Eckenhoff, J.E., et al. A new anesthetic agent - Forane: preliminary observations in man. Anesth. Analg. (Cleve.) 51: 439-447 (1972).

9. Snider, R.S., Neimer, W.T. A stereotaxic atlas of the cat brain. Univ. of Chicago Press (1961)

10. Julien, R.M., Kavan, E.M., Elliott, H.W. Effects of volatile anaesthetic agents on EEG activity recorded in limbic \& sensory systems. Canad. Anaesth. Soc. J. 19: 263-269 (1972).

11. Menkel, G., EGer, E.I. A comparative study of halothane and halopropane anesthesia. Including method for determining equipotency. Anesthesiology 24: 346-357 (1963).

12. Ecen, E.I., Saidisan, L.J., Brandstater, B. Minimum alveolar anesthetic concentration: a standard of anesthetic potency. Anesthesiology 26: 756-763 (1965).

13. Brazier, M.A.B. Role of the limbic system in maintenance of consciousness. Anesth. Analg. (Cleve.) 42: 748-751 (1963).

14. Albe-Fessard, D., Besson, J.M., Abdelmoumène, M. Action of anesthetics on somatic evoked activities. Internat. Anesth. Clin. 8: 129-164 (1970).

15. Julies, R.M., Kavan, E.M. Electrographic studies of a new volatile anaesthetic agent: Enflurane (Ethrane). J. Pharm. exp. Therap. 183: 393-403 (1972). 